\title{
Breeding Melon (Cucumis melo L.) for resistance to powdery mildew and fruit quality characteristics.
}

Abd Rabou, A. M. ${ }^{1}$; A.A. HAMED ${ }^{1}$; M, M. Ramadan ${ }^{2}$

Vegetables, Medicinal and Aromatic Plant Breeding Department, Hort. Res. Inst, Agricultural Research Center (ARC), Giza, Egypt.

Pests and Plant Protection Department, National Research Center(NRC) Giza, Egypt..

Corresponding author: aymanabdrabou40@gmail.com

\begin{abstract}
This study was conducted during the period from 2015 to 2017 to study resistance of powdery mildew in eight melon inbred lines and their hybrids in order to reduce losses in quality and yield. Eight different genetically inbred lines of melon were selfed for six generations to ensure homozygosity. They have been self-pollinated and intercrossed in a half diallel mating design to obtain 28 melon hybrids in 2015 . Thirty six melon genotypes ( 8 inbred Lines and 28 produced hybrids) were evaluated for their reaction to powdery mildew in 2016. The results showed that P5, P6, P7 and P8 of inbred lines, and all hybrids that contain P6 [(P1) X(P6), (P2) X(P6), (P3) $\mathrm{X}(\mathrm{P} 6)$, (P4) X(P6), (P5) X(P6), (P6) X(P7) and (P6) X(P8)] beside P5X P7, P5X P8 and P7X P8 showed low infection (between $1-12.5 \%$ ) that is meaning they are resistant. The genetic study showed that most hybrids appeared significant negative heterosis over its mid parent. On the contrary, most studied hybrids appeared significant positive heterosis over its better parent. The most potence ratio estimates indicated dominance towards powdery mildew resistance. In 2017 at WADY El-Natroon, The 36 hybrids were evaluated along with their parents to determine heterosis and potence ratio for the economic traits, viz., days to picking, early yield/plant, total yield/plant, average fruit weight, fruit shape index, netting, total soluble solids and flesh thickness. The results showed highly significant mean squares for all of the studied traits. Some crosses revealed highly significant and significant mid-parent and better-parent heterosis for many of studied traits. The inbred lines P5, P6 and P7 were resistant to powdery mildew and had good horticultural characteristics. They were the best and promising inbred lines.
\end{abstract}

Key words: Melon, Cucumis melo, Powdery Mildew, Heterosis, Potence ratio, Half Diallel.

\section{Introduction}

Melon $(2 \mathrm{n}=2 \mathrm{x}=24)$ is an important horticultural crop worldwide. Melon is one of the most economically important species of the cucurbitaceae family (Burger et al., 2003). it is divided into ssp. melo, which includes Western melon cultivars (Cantaloupe, Galia, Honeydew, Western shippers, Piel de Sapo, and Christmas melon) and ssp. agrestis, including wild types from India and Japan (Jeffrey 1980). A good genotype must have a high yield, market-standard size, good flesh thickness, small internal cavity, high flesh firmness and high soluble solids content (Nunes et al., 2005). In this sense, the choice of the best population in a study also depends on the goal of the program (Barros et al., 2011).

Heterosis breeding is one of the efficient tool of vegetables breeders to exploit the genetic diversity (Chaudhary and Pandey, 2010). Munger (1942) was the first one that observe hybrid vigour in melon. Pandey et al., (2005) showed that melon is enriched with great variability and therefore, heterosis breeding can be efficiently utilized to produce hybrids including fruit quality and high yield. Knowledge on the characters inheritance is useful to know strategy of breeding program (Feyzian et al., 2009). The improvement of fruit quality is a complex trait, as it includes external such as size, shape, and net, and internal fruit appearance such as color and taste. Heterosis in melon was investigated, but results were strictly dependent on parents and characters considered. Heterosis breeding is one of the most efficient tool of plant breeders to exploit the genetic diversity (Chaudhary and Pandey, 2010 and selim, 2019). Melon is enriched with great variability and heterosis breeding can be efficiently utilized to produce hybrids containing high yield and fruit quality (Pandey et al., 2005). José et al., (2005) reported that no heterosis for TSS concentration from negative to positive heterosis for fruit weight and diameter and a general positive heterosis for ovary shape, fruit shape and fruit length. Robinson et al., (1976) showed in melon that variability in fruit weight $(10 \mathrm{~g}-10 \mathrm{Kg})$, vine length (1-10 m), TSS (3-18\%) and flesh acidity (pH 3-7). Moreover, there is a need to develop suitable hybrids, which may be utilized on commercial scale (Dhaliwal, 1997).

Powdery mildew is a Very dangerous disease which reduces the yields of melon and other cucurbits, worldwide. Infection by powdery mildew is greatly influenced by the plant stage, humidity of air and temperature (Floris and Alvarez 1991). Singh (1987) reported that the fungi can sporulate and cause infection in very dry as well as wet atmosphere. Genotypes with useful genes and quantitative resistance to powdery mildew, independent of environment are of prime importance. Genetic control 
of resistance/tolerance to powdery mildew has been investigated using progeny resulting from crosses between susceptible and resistant/tolerant genotypes (Kenigsbuch and Cohen 1989). Perchepied et al. (2005) reported that sources of resistance to powdery mildew in melon have been identified, also the adult plant stage is usually the important stage for resistance screening, often seedlings were assessed for disease reactions under controlled conditions. There is no published study available on heterosis for resistance to powdery mildew in adult melon plants. The genetic relationship between resistance in adult greenhouse grown plants and adult field grown plants from the same parents could be used to develop elite resistant lines or to identify superior parents or crosses (Kadanapuradadoddi et al. 2011).

The present study was designed to assess the variation of $\mathrm{F} 1$ performances, potance ratio and heterosis for powdery mildew resistance on melon breeding programs pursue three main goals: (i) increase yield and earliness, (ii) improve fruit quality, and (iii) progress with powdery mildew resistances.

\section{Materials and Methods}

This study was carried out during spring seasons of the period from 2015 to 2017 in three different locations ( Kaha Vegetable Research Farm (KVRF), Kalubia; private farm, Wady EL-Natroon,
Behaira and Pests and Plant Protection Department, National Research Center Egypt). Eight local inbred lines of melon were used in this investigation (Table 1). These inbred lines were originated by the author of the present study from former melon breeding program by selfing and selection during 6 generations in KVRF. These inbred lines were cultivated at KVRF in February 2015 to increase inbred lines seeds by self pollination and produce crosses by half diallel method among the eight melon inbred lines in all possible combinations and obtain $28 \mathrm{~F} 1$ hybrids. The 28 hybrids were evaluated along with their parents $(8$ inbred lines) during spring season of 2016 and 2017 in the open field in private farm, Wady EL-Natroon, Behaira. Seeds of the genotypes were sown on $20^{\text {th }}$ February, 2016 and $25^{\text {th }}$ February, 2017, respectively, in foam trays under greenhouse. In a randomized complete block design with 3 replicates, seedlings were transplanted on $15^{\text {th }}$ March 2016 and $25^{\text {th }}$ March, 2017, respectively, in the open field. Thirty six genotypes (8 inbred lines, $28 \mathrm{~F} 1 \mathrm{~s}$ ) were planted in each replicate to horticultural characteristics evaluation. A single bed dimensions were $1.5 \mathrm{~m}$ width and $8.0 \mathrm{~m}$ length of each plot and the plants were sown at $50 \mathrm{~cm}$. Land preparation, fertilizer application and other field practices were conducted according to recommendations of the Egyptian Ministry of Agriculture.

Table 1. Performance of melon accessions used in the current study.

\begin{tabular}{lllll}
\hline Genotypes & Netting & shape index & cortex color & Flesh color \\
\hline P1 (13) & Smooth & Round & Yellow & Cream \\
P2 (14) & Smooth & Round & cream & Green \\
P3 (23) & Semi netting & oblong & Light Yellow & Orange \\
P4 (58) & Smooth & Round & cream & cream \\
P5 (64) & Netting & Round & Yellow & Green \\
P6 (66) & Netting & Round & Yellow & Orange \\
P7 (74) & Netting & Round & Yellow & Green \\
P8 (81) & Netting & cylindrical & Yellow & Orange \\
\hline
\end{tabular}

\section{- Evaluating for powdery mildew resistance}

Evaluating for powdery mildew resistance

for 36 genotypes ( 8 inbred lines and 28 produced hybrids) was carried out under greenhouse in a Department of Vegetables Crops, HRI, Dokki, Giza, during March 2016.

\section{Inoculation in the greenhouse}

The pathogen (Podosphaera xanthii) was isolated from a melon growers greenhouse at Department of Vegetables Crops, HRI, Dokki, Giza. A piece of powdery mildew infected melon leaf of size $1.5 \mathrm{~cm} \mathrm{X} 1.5 \mathrm{~cm}$ was stapled to the adaxial surface of leaves of all the test plants at 2-3 leaf stage. Stapled plants transferred to a growth chamber (50-70\% relative humidity, $23-25^{\circ} \mathrm{C}$ temperature and $12 / 12 \mathrm{~h}$ day/night). The second day, seedlings were brought back to the greenhouse benches. This method of inoculation was found ideal to initiate $P$. xanthii infection on abaxial surface of leaves in melon plants under the greenhouse conditions. Second inoculation was performed after three days of the first inoculation, to ensure uniform spread of disease on adaxial surface of leaves by breath blowing over powdery mildew infected cucumber leaves bearing freshly produced conidia. Immediately, inoculated plants were transferred to a growth chamber $(50-70 \%$ relative humidity, $23-25^{\circ} \mathrm{C}$ temperature and $12 / 12 \mathrm{~h}$ day/night). The next day, seedlings were brought back 
to the greenhouse surface. After second inoculation by 10-12 days, clear fungal growth was noticed on both sides of leaves of the inoculated plants. Symptoms first appeared as white or fluffy circular patches or spots on the under surface of the leaves and later spread to upper surface of leaves. Inoculated plants were maintained on greenhouse benches for 6 weeks. A single application of micronutrients at $0.5 \mathrm{ml} / \mathrm{l}$ of water was supplied at 2-3 leaf stage. Seedlings were hand watered daily. A nutrition solution containing $150 \mathrm{mg} \mathrm{N}, 150 \mathrm{mg}$ Pand $150 \mathrm{mg}$ K per liter of water was supplied every week (Kadanapuradadoddi et al 2011).

\section{Disease assessment:}

Infection on plants was measured 30 days after inoculation in the greenhouse. A linear 0-4 scale (Table 2) indicating average rating of all the leaves was used to assess the disease in experiment. On the basis of scoring of 3 individual leaves per plant and 10 plants in each genotype, Percent Disease Index (PDI) was calculated for each genotype using the formula proposed by (1969): $P D I=\frac{\text { Sum of numerical values }}{\text { No of lesaves } x \text { Maximum rating }} \times 100$

Table 2. Powdery mildew disease severity estimating into 5 categories according to James (1971).

\begin{tabular}{llll}
\hline Scale & Mildew covering of leaf surface & symptoms & Reaction \\
\hline $\mathbf{0}$ & $0 \%$ & No symptoms of infection & High resistance (HR) \\
$\mathbf{1}$ & $1-12.5 \%$ & Very weak infection & Resistance (R) \\
$\mathbf{2}$ & $13-25 \%$ & Weak infection & Tolerance (T) \\
$\mathbf{3}$ & $25.5-50 \%$ & Moderate infection & Susceptible (S) \\
$\mathbf{4}$ & $50.5-100 \%$ & Very severe infection & High susceptible (HS) \\
\hline
\end{tabular}

\section{- Evaluation for horticultural characteristics}

The horticultural evaluation of the 8 inbred lines and 28 produced hybrids was carried out in private farm, Wady EL-Natroon, Behaira, , Egypt. during spring season in 2016 and 2017. Data were recorded as follows:

1. Days to picking: number of days from cultivation to start pickling.

2. Early yield/plant $(\mathrm{kg})$ : Total fruit weight of the first two pickings.

3. Total yield per plant $(\mathrm{Kg})$ : Total yield per plant was determined by weighting all produced fruits per plant.

4. Average fruit weight $(\mathrm{Kg})$ : Average fruit weight was determined as average weight of 10 fruits randomly taken from each genotypes in the $3^{\text {th }}$ harvest.

5. Fruit shape index: Shape index computed as the ratio of fruit length to fruit diameter, each genotypes was represented by 10 fruits. The scale of fruit shape (table 3) divided fruits into 4 categories as showing in Table 3 .

6. Netting (\%): The netting was estimated as a proportion of the netting coated fruit cortex to full fruit cortex as optical manner and calculated as the average of 10 fruits randomly taken from each genotypes.

7. Total soluble solids (TSS): It was measured in 10 yellow-ripe fruits of each genotypes utilizing a hand refractometer.

8. Flesh thickness (cm.): flesh thickness were measured as the average of 10 fruits randomly taken from each genotype by rule.

Table 3. Scale of fruit shape index of melon fruits (Rashidi and Seyfi 2007).

\begin{tabular}{ll}
\hline Range & Shape \\
\hline$>0.88$ & oblate \\
0.88 to 1.1 & round \\
1.1 to 1.5 & cylindrical \\
$<1.5$ & oblong \\
\hline
\end{tabular}

Collected data were statistically analyzed and mean comparisons were depend on the LSD test as reported by Gomez and Gomez (1984). Heterosis over mid parent and better parent was estimated as percentage after calculating heterosis of respective parent by utilizing formula as reported by Falconer and Mackay (1996). Potence ratio was estimated by utilizing formula as reported by Smith (1952).

\section{Results and Discussion}

Evaluating for powdery mildew resistance under artificial infection

Data obtained on the reaction of melon inbred lines to powdery mildew under artificial infection conditions on greenhouse during March 2016 are presented in Table 4 and Fig 1. Among 36 
genotypes ( 8 inbred lines and 28 produced hybrids) and according the scale in table 2, genotypes P5, P6, $\mathrm{P} 7$ and P8 of inbred lines and the line (P6) was common to all resistant hybrids $[(\mathrm{P} 1) \mathrm{X}(\mathrm{P} 6),(\mathrm{P} 2)$ $\mathrm{X}(\mathrm{P} 6)$, (P3) X(P6), (P4) X(P6), (P5) X(P6), (P6) $\mathrm{X}(\mathrm{P} 7)$ and (P6) X(P8)] beside P5X P7, P5X P8 and P7X P8 showed low infection (between 1-12.5\%) that is meaning they are resistant. On the other hand, P1, P2, P3 and P4 showed moderate infection and very severe infection, these genotypes are considered susceptible and high susceptible (between 25.5 $100 \%$ ) to powdery mildew. While, the other hybrids were tolerant, susceptible and highly susceptible. These results agreement with EL- Sayed et al (2019) who studied 10 different inbred lines of melon for their response to powdery mildew and found, plants of the inbred lines No.1,2,3,5,6,7,8,9 and 10 showed clear symptoms of infection and low disease severity to powdery mildew and plants of line (4) showed high resistance to powdery mildew.

Table 4. The reaction of melon genotypes evaluated for powdery mildew resistance under artificial infection conditions in 2016 season.

\begin{tabular}{|c|c|c|}
\hline Genotypes & Disease severity $(\%)$ & Reaction \\
\hline P1 (13) & 89 & $\mathrm{HS}$ \\
\hline P2 (14) & 89.7 & HS \\
\hline P3 (23) & 94.3 & HS \\
\hline P4 (58) & 47.2 & S \\
\hline P5 (64) & 5.3 & $\mathrm{R}$ \\
\hline P6 (66) & 4.4 & $\mathrm{R}$ \\
\hline P7 (74) & 4.9 & $\mathrm{R}$ \\
\hline P8 (81) & 4.2 & $\mathrm{R}$ \\
\hline P1X P2 & 87.9 & HS \\
\hline P1X P3 & 91.7 & HS \\
\hline P1X P4 & 92.3 & HS \\
\hline P1X P5 & 52.2 & HS \\
\hline P1X P6 & 12.3 & $\mathrm{R}$ \\
\hline P1X P7 & 15.2 & $\mathrm{~T}$ \\
\hline P1X P8 & 17.5 & $\mathrm{~T}$ \\
\hline P2X P3 & 93.2 & HS \\
\hline P2X P4 & 87.2 & HS \\
\hline P2X P5 & 56.5 & HS \\
\hline P2X P6 & 14.1 & $\mathrm{R}$ \\
\hline P2X P7 & 22.2 & $\mathrm{~T}$ \\
\hline P2X P8 & 29.4 & $\mathrm{~S}$ \\
\hline P3X P4 & 66.3 & HS \\
\hline P3X P5 & 16.2 & $\mathrm{~T}$ \\
\hline P3X P6 & 13.2 & $\mathrm{R}$ \\
\hline P3X P7 & 20.3 & $\mathrm{~T}$ \\
\hline P3X P8 & 13.2 & $\mathrm{~T}$ \\
\hline P4X P5 & 32.2 & $S$ \\
\hline P4X P6 & 10.1 & $\mathrm{R}$ \\
\hline P4X P7 & 38.2 & S \\
\hline P4X P8 & 17.3 & $\mathrm{~T}$ \\
\hline P5X P6 & 2.2 & $\mathrm{R}$ \\
\hline P5X P7 & 7.2 & $\mathrm{R}$ \\
\hline P5X P8 & 8.5 & $\mathrm{R}$ \\
\hline P6X P7 & 3.2 & $\mathrm{R}$ \\
\hline P6X P8 & 5 & $\mathrm{R}$ \\
\hline P7X P8 & 12.1 & $\mathrm{R}$ \\
\hline LSD & 2.7 & \\
\hline
\end{tabular}

$\mathrm{HR}=$ Highly resistant (0), $\mathrm{R}=$ Resistant (1-12.5), $\mathrm{T}=$ Tolerant (13-25), HS= Highly susceptible (25.5-50), $\mathrm{S}=\mathrm{Susceptible} \mathrm{(50.5-100).}$

\section{Genetic study to powdery mildew resistance}

Concerning heterosis, 16 out of the 28 evaluated hybrids exhibited significant negative heterosis over its mid-parent. Meanwhile, 3 out of the 28 evaluated hybrids exhibited significant negative heterosis over its better parent for powdery mildew. The potence ratio estimates supported the data on heterosis. The potence ratio estimates indicated dominance towards the resistance for powdery mildew in 13 crosses, value 8 crosses of them gave 
potence ratio values less than one $(\mathrm{P}<-1)$, refer to Partial dominance of powdery mildew resistance character towards the resistant parent in this crosses and the others were over than one $(\mathrm{P}>-1)$, refer to partial dominance towards resistant parent. Meanwhile, the remaining crosses gave partial or complete dominance towards the susceptible parent for powdery mildew.

Table 5. Quantitative genetic parameters of powdery mildew in melon genotypes

\begin{tabular}{|c|c|c|c|}
\hline Genotypes & Potance ratio & MPH (\%) & $\mathrm{BPH}(\%)$ \\
\hline P1X P2 & 4.14 & $-2 \%$ & $-1 \%$ \\
\hline P1X P3 & -0.02 & $0 \%$ & $3 \%$ \\
\hline P1X P4 & -1.16 & $36 \%$ & $96 \%$ \\
\hline P1X P5 & -0.12 & $11 \%$ & $885 \%$ \\
\hline P1X P6 & -0.81 & $-74 \%$ & $180 \%$ \\
\hline P1X P7 & 0.76 & $-68 \%$ & $210 \%$ \\
\hline P1X P8 & 0.69 & $-62 \%$ & $317 \%$ \\
\hline P2X P3 & 0.52 & $1 \%$ & $4 \%$ \\
\hline P2X P4 & -0.88 & $27 \%$ & $85 \%$ \\
\hline P2X P5 & -0.72 & $115 \%$ & $966 \%$ \\
\hline P2X P6 & -0.77 & $-70 \%$ & $220 \%$ \\
\hline P2X P7 & 0.59 & $-53 \%$ & $353 \%$ \\
\hline P2X P8 & 0.41 & $-37 \%$ & $600 \%$ \\
\hline P3X P4 & 0.19 & $-6 \%$ & $40 \%$ \\
\hline P3X P5 & 0.76 & $-67 \%$ & $206 \%$ \\
\hline P3X P6 & 0.80 & $-73 \%$ & $200 \%$ \\
\hline P3X P7 & 0.66 & $-59 \%$ & $314 \%$ \\
\hline P3X P8 & 0.80 & $-73 \%$ & $214 \%$ \\
\hline P4X P5 & -0.28 & $23 \%$ & $508 \%$ \\
\hline P4X P6 & 0.73 & $-61 \%$ & $130 \%$ \\
\hline P4X P7 & -0.57 & $47 \%$ & $680 \%$ \\
\hline P4X P8 & 15.27 & $-33 \%$ & $312 \%$ \\
\hline P5X P6 & 5.89 & $-55 \%$ & $-50 \%$ \\
\hline P5X P7 & -10.50 & $41 \%$ & $47 \%$ \\
\hline P5X P8 & -6.82 & $79 \%$ & $102 \%$ \\
\hline P6X P7 & 5.80 & $-31 \%$ & $-27 \%$ \\
\hline P6X P8 & -7.00 & $16 \%$ & $19 \%$ \\
\hline P7X P8 & -21.57 & $166 \%$ & $188 \%$ \\
\hline
\end{tabular}

$\mathrm{MPH}=$ Mid parent heterosis, $\mathrm{BPH}=$ Bitter parent heterosis 

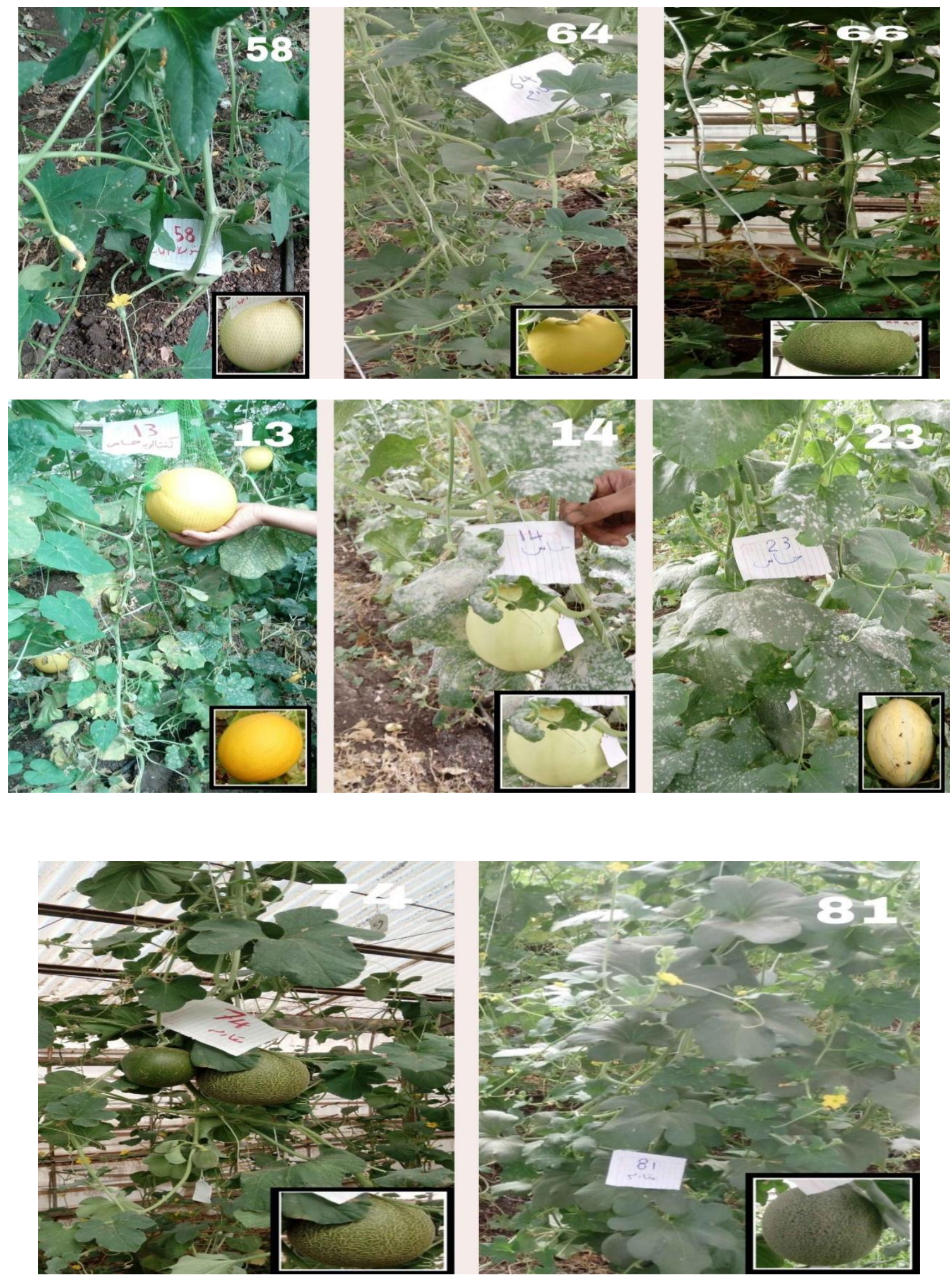

Fig. 1: powdery mildew resistance and Fruit quality differences of the parental melon genotypes. 


\section{Evaluation for horticultural characteristics}

The ANOVA for the design (Table 6) revealed significant differences between all genotypes for all the studied characters. Total variation among hybrids indicated that parents selected for the present study were genetically divergent.

Days to picking

Data showed that P4 had the lowest number of days to picking with P5 and P6 in 2016 and alone in 2017 with significantly different from other inbred lines. On the other hand, the results recorded that P2 had the highest number days to picking with significant differences between the other inbred lines in the two seasons. Also, the results revealed that the hybrids P1X P4, P1X P6, P2X P6, P3X P6, P5X P6, P6X P7 and P6X P8 gave the lowest number of days to picking in both seasons compared with the other hybrids.

\section{Early Yield/plant (kg)}

Obtained data on early yield in the spring seasons of 2016 and 2017 were shown in Table (6). P4 had the greatest early yield and was significantly different over all other evaluated inbred lines. On the other hand, the results recorded that the parents $\mathrm{P} 2 \mathrm{had}$ $0.00 \mathrm{~kg}$ early yield in the two seasons. Also, the results revealed that the hybrids $\mathrm{P} 3 \mathrm{XP} 4, \mathrm{P} 3 \mathrm{XP} 6$ and P4XP6 gave the highest early yield/ plant in both seasons compared with the other hybrids with significantly with the hybrids.

\section{Total Yield/plant $(\mathrm{kg})$}

The greatest total yield was shown in P3 and P6 with significantly different over all other melon inbred lines. Additionally, P1XP6, P3XP6 and P4XP6 had greatest total yield in both seasons compared with the other hybrids with significantly with the hybrids. In contrast, P3 had the least total yield with significant differences with other inbred lines. Also, P7XP8 had the least total yield with significant differences from other hybrids in both seasons.

\section{Fruit quality}

Obtained data on average fruit weight, fruit shape index, total soluble solids (\%), flesh thickness and netting (\%) in the spring seasons of 2016 and 2017 were illustrated in Table 6 and Fig 1.

\section{a- Average fruit weigh}

Regarding average fruit weight, P3 gave the highest average fruit weight as inbred lines and was significantly different from whole the others. P4 sorted second but it wasn't significantly different from P7 and P8. The least average fruit weight was shown in P5 but it wasn't significantly different from P1, P2 and P6 in both seasons. Also the results refer that the hybrid P3X P4 and P7X P8 in the both seasons, with significantly different from whole the others.

b- Fruit shape index

Concerning fruit shape index, P3 had the highest fruit shape index with significant different over all other parents. Also, P8 ranked second in this trait. The other parents were shown standard marketing in fruit shape index (0.8-1.1). All hybrids which contain P3 were the highest fruit shape index with significant different over all other evaluated. According to scale in table 3 , the round shape is the marketing fruit, so that all parents and hybrids which ranged from 0.8 to 1.1 were the marketing fruit.

c- Total soluble solids (\%)

In respect to total soluble solids (TSS), P6 had the highest TSS with significant different from all inbred lines. Additionally, P5 and P7 ranked second in this trait with significant difference from the other evaluated inbred lines. Most of produced hybrids which contain P6 and P5X P8 were the highest fruit shape index with significant different over all other evaluated. On the other hand, P3 and P8 had the lowest TSS with significant different from other inbred lines. Also, most of produced hybrids which contain P3 and P8 were the lowest TSS with significant different over all other evaluated hybrids.

d- Flesh thickness

Inbred lines regarding flesh thickness, P6 and P5 had the greatest flesh thickness with significant difference over other inbred lines. In contrast, the narrowest flesh thickness was shown in P3 and P8 with significant difference from over other inbred lines.

P1X P5, P1X P6, P3X P6, P4X P5, P4X P6 and P5X P6 were greatest flesh thickness with significant different over all other evaluated hybrids. On the other hand, most of produced hybrids which contain P3 and P8 were the narrowest flesh thickness with significant different over all other evaluated hybrids.

\section{e- Netting (\%)}

In respect to netting percentage, $\mathrm{P} 5, \mathrm{P} 6$ and $\mathrm{P} 7$ had the highest netting percentage with significant differences from all evaluated inbred lines. The least netting percentage was shown in $\mathrm{P} 1, \mathrm{P} 2$ and $\mathrm{P} 4$ with significant different from $\mathrm{P} 8$ and $\mathrm{P} 3$.

Most of produced hybrids which contain P5, P6 and P7 were the highest netting percentage with significant different over all other evaluated hybrids. On the other hand, the crossing between P1, P2 and P4 had the least netting percentage with significant different over all other evaluated hybrids.

The significant differences resulted among parents and crosses are in agreement with the results reported by Chandha et al. (2001). 
Table 6. Yield component and fruit quality of melon genotypes evaluated in 2016 and 2017.

\begin{tabular}{|c|c|c|c|c|c|c|c|c|c|c|c|c|c|c|c|c|}
\hline \multirow[t]{2}{*}{$\begin{array}{l}\text { Genoty } \\
\text { pes }\end{array}$} & \multirow{2}{*}{$\begin{array}{l}\begin{array}{l}\text { Average } \\
\text { weight }(\mathrm{g})\end{array} \\
2016\end{array}$} & fruit & \multicolumn{2}{|c|}{$\begin{array}{l}\text { Days } \\
\text { picking }\end{array}$} & \multicolumn{2}{|c|}{$\begin{array}{l}\text { Total } \\
\text { yield/plant (kg) }\end{array}$} & \multicolumn{2}{|c|}{$\begin{array}{l}\text { Early yield/plant } \\
\text { (Kg) }\end{array}$} & \multicolumn{2}{|c|}{ Netting \% } & \multicolumn{2}{|c|}{$\begin{array}{l}\text { Total soluble } \\
\text { solids (\%) }\end{array}$} & \multicolumn{2}{|c|}{$\begin{array}{l}\text { Flesh } \\
\text { thickness } \\
\text { (cm) }\end{array}$} & \multicolumn{2}{|c|}{$\begin{array}{l}\text { Shape } \\
\text { index }\end{array}$} \\
\hline & & 2017 & $\begin{array}{l}201 \\
6\end{array}$ & $\begin{array}{l}201 \\
7\end{array}$ & 2016 & 2017 & 2016 & 2017 & $\begin{array}{l}20 \\
16\end{array}$ & $\begin{array}{l}\mathbf{2 0} \\
\mathbf{1 7}\end{array}$ & 2016 & 2017 & $\begin{array}{l}20 \\
16\end{array}$ & $\begin{array}{l}20 \\
17\end{array}$ & $\begin{array}{l}20 \\
16\end{array}$ & $\begin{array}{l}\mathbf{2 0} \\
\mathbf{1 7}\end{array}$ \\
\hline P1 (13) & 0.75 & 0.87 & $\begin{array}{l}113 . \\
48\end{array}$ & $\begin{array}{l}114 . \\
98\end{array}$ & 2.75 & 2.98 & 0.75 & 0.88 & $\mathbf{0}$ & $\mathbf{0}$ & 11.11 & 11.46 & $\begin{array}{l}6.0 \\
4\end{array}$ & $\begin{array}{l}5.8 \\
5\end{array}$ & $\begin{array}{l}0.9 \\
0\end{array}$ & $\begin{array}{l}1.0 \\
1\end{array}$ \\
\hline P2 (14) & 0.88 & 1.00 & $\begin{array}{l}129 . \\
03\end{array}$ & $\begin{array}{l}127 . \\
53\end{array}$ & 2.90 & 3.15 & 0.00 & 0.00 & $\mathbf{0}$ & 0 & 10.21 & 10.16 & $\begin{array}{l}4.0 \\
2\end{array}$ & $\begin{array}{l}3.8 \\
5\end{array}$ & $\begin{array}{l}1.1 \\
0\end{array}$ & $\begin{array}{l}1.0 \\
1\end{array}$ \\
\hline P3 (23) & 1.65 & 1.87 & $\begin{array}{l}112 . \\
23\end{array}$ & $\begin{array}{l}110 . \\
73\end{array}$ & 4.64 & 4.59 & 1.42 & 1.29 & $\begin{array}{l}28 . \\
6\end{array}$ & $\begin{array}{l}30, \\
6\end{array}$ & 9.32 & 9.46 & $\begin{array}{l}6.1 \\
0\end{array}$ & $\begin{array}{l}5.8 \\
5\end{array}$ & $\begin{array}{l}1.9 \\
0\end{array}$ & $\begin{array}{l}2.0 \\
1\end{array}$ \\
\hline P4 (58) & 1.22 & 1.44 & $\begin{array}{l}105 . \\
98\end{array}$ & $\begin{array}{l}102 . \\
48\end{array}$ & 3.86 & 3.63 & 1.85 & 1.72 & 0 & 0 & 11.14 & 11.16 & $\begin{array}{l}6.0 \\
2\end{array}$ & $\begin{array}{l}6.1 \\
5\end{array}$ & $\begin{array}{l}0.9 \\
0\end{array}$ & $\begin{array}{l}1.0 \\
3\end{array}$ \\
\hline P5 (64) & 0.70 & 0.82 & $\begin{array}{l}106 . \\
39\end{array}$ & $\begin{array}{l}107 . \\
89\end{array}$ & 2.49 & 2.74 & 1.31 & 1.44 & $\begin{array}{l}10 \\
0\end{array}$ & $\begin{array}{l}10 \\
0\end{array}$ & 13.80 & 14.26 & $\begin{array}{l}6.4 \\
0\end{array}$ & $\begin{array}{l}6.5 \\
5\end{array}$ & $\begin{array}{l}1.1 \\
0\end{array}$ & $\begin{array}{l}1.0 \\
4\end{array}$ \\
\hline P6 (66) & 0.82 & 0.94 & $\begin{array}{l}106 . \\
72\end{array}$ & $\begin{array}{l}111 . \\
22\end{array}$ & 4.57 & 4.80 & 1.45 & 1.58 & $\begin{array}{l}10 \\
0\end{array}$ & $\begin{array}{l}10 \\
0\end{array}$ & 15.30 & 15.76 & $\begin{array}{l}6.5 \\
0\end{array}$ & $\begin{array}{l}6.6 \\
5\end{array}$ & $\begin{array}{l}0.9 \\
5\end{array}$ & $\begin{array}{l}0.8 \\
9\end{array}$ \\
\hline P7 (74) & 1.05 & 1.17 & $\begin{array}{l}112 . \\
80\end{array}$ & $\begin{array}{l}111 . \\
30\end{array}$ & 2.14 & 1.91 & 0.93 & 0.80 & $\begin{array}{l}10 \\
0\end{array}$ & $\begin{array}{l}10 \\
0\end{array}$ & 13.10 & 13.26 & $\begin{array}{l}6.0 \\
0\end{array}$ & $\begin{array}{l}6.1 \\
3\end{array}$ & $\begin{array}{l}1.0 \\
0\end{array}$ & $\begin{array}{l}0.9 \\
4\end{array}$ \\
\hline P8 (81) & 1.12 & 1.34 & $\begin{array}{l}109 . \\
90\end{array}$ & $\begin{array}{l}111 . \\
40\end{array}$ & 3.57 & 3.80 & 1.20 & 1.33 & $\begin{array}{l}68 . \\
1\end{array}$ & $\begin{array}{l}62 . \\
7\end{array}$ & 9.25 & 9.46 & $\begin{array}{l}5.5 \\
0\end{array}$ & $\begin{array}{l}5.6 \\
3\end{array}$ & $\begin{array}{l}1.3 \\
0\end{array}$ & $\begin{array}{l}1.4 \\
6\end{array}$ \\
\hline P1X P2 & 0.95 & 1.07 & $\begin{array}{l}113 . \\
25\end{array}$ & $\begin{array}{l}110 . \\
75\end{array}$ & 3.76 & 3.53 & 1.15 & 1.02 & 0 & 0 & 11.50 & 11.66 & $\begin{array}{l}4.5 \\
0\end{array}$ & $\begin{array}{l}4.6 \\
7\end{array}$ & $\begin{array}{l}1.0 \\
0\end{array}$ & $\begin{array}{l}0.9 \\
4\end{array}$ \\
\hline P1X P3 & 1.12 & 1.34 & $\begin{array}{l}114 . \\
77\end{array}$ & $\begin{array}{l}118 . \\
27\end{array}$ & 3.76 & 3.99 & 1.07 & 1.20 & $\begin{array}{l}12 . \\
4\end{array}$ & $\begin{array}{l}17 . \\
6\end{array}$ & 10.50 & 10.66 & $\begin{array}{l}6.1 \\
0\end{array}$ & $\begin{array}{l}6.2 \\
5\end{array}$ & $\begin{array}{l}1.5 \\
0\end{array}$ & $\begin{array}{l}1.4 \\
4\end{array}$ \\
\hline P1X P4 & 0.97 & 1.09 & $\begin{array}{l}105 . \\
93\end{array}$ & $\begin{array}{l}105 . \\
43\end{array}$ & 3.95 & 3.72 & 0.87 & 1.00 & 0 & 0 & 12.36 & 12.46 & $\begin{array}{l}6.1 \\
0\end{array}$ & $\begin{array}{l}6.2 \\
3\end{array}$ & $\begin{array}{l}0.9 \\
0\end{array}$ & $\begin{array}{l}0.8 \\
4\end{array}$ \\
\hline P1X P5 & 0.81 & 0.93 & $\begin{array}{l}111 . \\
09\end{array}$ & $\begin{array}{l}108 . \\
59\end{array}$ & 3.88 & 4.11 & 0.81 & 0.96 & $\begin{array}{l}10 \\
0\end{array}$ & $\begin{array}{l}10 \\
0\end{array}$ & 13.50 & 13.66 & $\begin{array}{l}6.4 \\
5\end{array}$ & $\begin{array}{l}6.5 \\
7\end{array}$ & $\begin{array}{l}1.0 \\
0\end{array}$ & $\begin{array}{l}0.9 \\
4\end{array}$ \\
\hline P1X P6 & 0.83 & 0.95 & $\begin{array}{l}107 . \\
08\end{array}$ & $\begin{array}{l}108 . \\
58\end{array}$ & 4.97 & 4.52 & 0.83 & 0.98 & $\begin{array}{l}10 \\
0\end{array}$ & $\begin{array}{l}10 \\
0\end{array}$ & 16.66 & 16.76 & $\begin{array}{l}6.7 \\
0\end{array}$ & $\begin{array}{l}6.8 \\
2\end{array}$ & $\begin{array}{l}1.0 \\
0\end{array}$ & $\begin{array}{l}0.9 \\
8\end{array}$ \\
\hline P1X P7 & 1.15 & 1.37 & $\begin{array}{l}110 . \\
05\end{array}$ & $\begin{array}{l}108 . \\
55\end{array}$ & 4.46 & 4.23 & 1.65 & 1.78 & $\begin{array}{l}10 \\
0\end{array}$ & $\begin{array}{l}10 \\
0\end{array}$ & 13.06 & 13.46 & $\begin{array}{l}6.2 \\
0\end{array}$ & $\begin{array}{l}6.3 \\
3\end{array}$ & $\begin{array}{l}0.9 \\
5\end{array}$ & $\begin{array}{l}0.9 \\
3\end{array}$ \\
\hline P1X P8 & 0.95 & 1.07 & $\begin{array}{l}107 . \\
90\end{array}$ & $\begin{array}{l}105 . \\
40\end{array}$ & 3.20 & 3.45 & 0.70 & 0.83 & $\begin{array}{l}50 . \\
9\end{array}$ & $\begin{array}{l}43 . \\
7\end{array}$ & 10.47 & 11.22 & $\begin{array}{l}5.7 \\
0\end{array}$ & $\begin{array}{l}5.8 \\
2\end{array}$ & $\begin{array}{l}1.1 \\
0\end{array}$ & $\begin{array}{l}1.0 \\
8\end{array}$ \\
\hline P2X P3 & 1.40 & 1.62 & $\begin{array}{l}109 . \\
43\end{array}$ & $\begin{array}{l}107 . \\
93\end{array}$ & 3.82 & 4.05 & 1.70 & 1.85 & $\begin{array}{l}25 . \\
0\end{array}$ & $\begin{array}{l}29 . \\
6\end{array}$ & 9.65 & 10.06 & $\begin{array}{l}4.8 \\
0\end{array}$ & $\begin{array}{l}4.9 \\
1\end{array}$ & $\begin{array}{l}1.4 \\
0\end{array}$ & $\begin{array}{l}1.5 \\
1\end{array}$ \\
\hline P2X P4 & 0.94 & 1.06 & $\begin{array}{l}105 . \\
20\end{array}$ & $\begin{array}{l}103 . \\
70\end{array}$ & 3.56 & 3.33 & 0.74 & 0.61 & 0 & 0 & 12.78 & 12.46 & $\begin{array}{l}5.6 \\
0\end{array}$ & $\begin{array}{l}5.7 \\
4\end{array}$ & $\begin{array}{l}1.0 \\
0\end{array}$ & $\begin{array}{l}1.1 \\
0\end{array}$ \\
\hline P2X P5 & 0.79 & 0.85 & $\begin{array}{l}108 . \\
84\end{array}$ & $\begin{array}{l}106 . \\
34\end{array}$ & 2.42 & 2.65 & 1.01 & 1.14 & $\begin{array}{l}10 \\
0\end{array}$ & $\begin{array}{l}10 \\
0\end{array}$ & 13.90 & 14.36 & $\begin{array}{l}5.9 \\
0\end{array}$ & $\begin{array}{l}5.9 \\
4\end{array}$ & $\begin{array}{l}1.2 \\
0\end{array}$ & $\begin{array}{l}1.1 \\
4\end{array}$ \\
\hline P2X P6 & 0.89 & 0.97 & $\begin{array}{l}105 . \\
41\end{array}$ & $\begin{array}{l}104 . \\
91\end{array}$ & 3.99 & 4.24 & 0.79 & 0.94 & $\begin{array}{l}10 \\
0\end{array}$ & $\begin{array}{l}10 \\
0\end{array}$ & 14.50 & 14.96 & $\begin{array}{l}5.7 \\
0\end{array}$ & $\begin{array}{l}5.8 \\
4\end{array}$ & $\begin{array}{l}1.1 \\
0\end{array}$ & $\begin{array}{l}0.9 \\
4\end{array}$ \\
\hline P2X P7 & 1.10 & 1.22 & $\begin{array}{l}107 . \\
25\end{array}$ & $\begin{array}{l}110 . \\
75\end{array}$ & 2.79 & 2.56 & 0.99 & 1.12 & $\begin{array}{l}10 \\
0\end{array}$ & $\begin{array}{l}10 \\
0\end{array}$ & 13.50 & 13.34 & $\begin{array}{l}4.9 \\
0\end{array}$ & $\begin{array}{l}5.0 \\
2\end{array}$ & $\begin{array}{l}1.1 \\
0\end{array}$ & $\begin{array}{l}1.0 \\
4\end{array}$ \\
\hline P2X P8 & 1.06 & 1.18 & $\begin{array}{l}112 . \\
80\end{array}$ & $\begin{array}{l}111 . \\
30\end{array}$ & 3.20 & 3.43 & 1.06 & 0.93 & $\begin{array}{l}40 . \\
7\end{array}$ & $\begin{array}{l}48 . \\
6\end{array}$ & 11.30 & 11.14 & $\begin{array}{l}5.7 \\
0\end{array}$ & $\begin{array}{l}5.8 \\
5\end{array}$ & $\begin{array}{l}1.2 \\
0\end{array}$ & $\begin{array}{l}1.3 \\
1\end{array}$ \\
\hline P3X P4 & 1.75 & 1.87 & $\begin{array}{l}109 . \\
07\end{array}$ & $\begin{array}{l}107 . \\
57\end{array}$ & 4.39 & 4.62 & 1.95 & 1.98 & $\begin{array}{l}33 . \\
6\end{array}$ & $\begin{array}{l}30 . \\
1\end{array}$ & 9.40 & 9.86 & $\begin{array}{l}6.1 \\
0\end{array}$ & $\begin{array}{l}6.2 \\
4\end{array}$ & $\begin{array}{l}1.5 \\
0\end{array}$ & $\begin{array}{l}1.6 \\
4\end{array}$ \\
\hline P3X P5 & 0.83 & 0.95 & $\begin{array}{l}113 . \\
16\end{array}$ & $\begin{array}{l}114 . \\
66\end{array}$ & 3.31 & 3.56 & 0.83 & 0.98 & $\begin{array}{l}10 \\
0\end{array}$ & $\begin{array}{l}10 \\
0\end{array}$ & 10.50 & 10.66 & $\begin{array}{l}6.3 \\
0\end{array}$ & $\begin{array}{l}6.4 \\
3\end{array}$ & $\begin{array}{l}1.6 \\
0\end{array}$ & $\begin{array}{l}1.7 \\
1\end{array}$ \\
\hline P3X P6 & 0.92 & 0.84 & $\begin{array}{l}104 . \\
68\end{array}$ & $\begin{array}{l}105 . \\
18\end{array}$ & 4.87 & 4.74 & 1.80 & 1.93 & $\begin{array}{l}10 \\
0\end{array}$ & $\begin{array}{l}10 \\
0\end{array}$ & 15.60 & 16.06 & $\begin{array}{l}6.5 \\
0\end{array}$ & $\begin{array}{l}6.6 \\
4\end{array}$ & $\begin{array}{l}1.3 \\
0\end{array}$ & $\begin{array}{l}1.5 \\
1\end{array}$ \\
\hline P3X P7 & 1.18 & 1.30 & $\begin{array}{l}110 . \\
01\end{array}$ & $\begin{array}{l}113 . \\
51\end{array}$ & 3.28 & 3.53 & 0.92 & 1.07 & $\begin{array}{l}10 \\
0\end{array}$ & $\begin{array}{l}10 \\
0\end{array}$ & 12.40 & 12.56 & $\begin{array}{l}5.9 \\
0\end{array}$ & $\begin{array}{l}6.0 \\
3\end{array}$ & $\begin{array}{l}1.5 \\
0\end{array}$ & $\begin{array}{l}1.4 \\
4\end{array}$ \\
\hline P3X P8 & 1.40 & 1.42 & $\begin{array}{l}109 . \\
80\end{array}$ & $\begin{array}{l}107 . \\
30\end{array}$ & 4.20 & 4.43 & 1.67 & 1.80 & $\begin{array}{l}77 . \\
5\end{array}$ & $\begin{array}{l}71 . \\
7\end{array}$ & 9.20 & 9.66 & $\begin{array}{l}5.6 \\
0\end{array}$ & $\begin{array}{l}5.4 \\
5\end{array}$ & $\begin{array}{l}2.1 \\
0\end{array}$ & $\begin{array}{l}2.1 \\
4\end{array}$ \\
\hline P4X P5 & 1.40 & 1.52 & $\begin{array}{l}110 . \\
84\end{array}$ & $\begin{array}{l}114 . \\
34\end{array}$ & 4.10 & 3.87 & 1.24 & 1.39 & $\begin{array}{l}10 \\
0\end{array}$ & $\begin{array}{l}10 \\
0\end{array}$ & 12.60 & 13.06 & $\begin{array}{l}6.4 \\
2\end{array}$ & $\begin{array}{l}6.5 \\
5\end{array}$ & $\begin{array}{l}1.1 \\
0\end{array}$ & $\begin{array}{l}1.0 \\
4\end{array}$ \\
\hline P4X P6 & 0.89 & 1.01 & $\begin{array}{l}104 . \\
22\end{array}$ & $\begin{array}{l}105 . \\
72\end{array}$ & 4.68 & 4.53 & 1.96 & 1.89 & $\begin{array}{l}10 \\
0\end{array}$ & $\begin{array}{l}10 \\
0\end{array}$ & 13.90 & 14.06 & $\begin{array}{l}6.7 \\
0\end{array}$ & $\begin{array}{l}6.8 \\
3\end{array}$ & $\begin{array}{l}0.8 \\
7\end{array}$ & $\begin{array}{l}0.8 \\
1\end{array}$ \\
\hline P4X P7 & 0.97 & 1.09 & $\begin{array}{l}114 . \\
92\end{array}$ & $\begin{array}{l}112 . \\
42\end{array}$ & 3.75 & 3.98 & 0.86 & 1.01 & $\begin{array}{l}10 \\
0\end{array}$ & $\begin{array}{l}10 \\
0\end{array}$ & 13.90 & 14.06 & $\begin{array}{l}6.3 \\
0\end{array}$ & $\begin{array}{l}6.4 \\
7\end{array}$ & $\begin{array}{l}1.0 \\
0\end{array}$ & $\begin{array}{l}1.0 \\
6\end{array}$ \\
\hline P4X P8 & 1.24 & 1.02 & $\begin{array}{l}107 . \\
80\end{array}$ & $\begin{array}{l}109 . \\
30\end{array}$ & 3.20 & 3.45 & 1.14 & 1.27 & $\begin{array}{l}68 . \\
1\end{array}$ & 69 & 9.30 & 9.46 & $\begin{array}{l}5.8 \\
\text { 0 }\end{array}$ & $\begin{array}{l}5.9 \\
3\end{array}$ & $\begin{array}{l}1.2 \\
0\end{array}$ & $\begin{array}{l}1.2 \\
6\end{array}$ \\
\hline P5X P6 & 0.86 & 0.98 & $\begin{array}{l}105 . \\
55\end{array}$ & $\begin{array}{l}104 . \\
05\end{array}$ & 3.77 & 4.00 & 0.99 & 1.14 & $\begin{array}{l}10 \\
0\end{array}$ & $\begin{array}{l}10 \\
0\end{array}$ & 16.90 & 17.36 & $\begin{array}{l}6.5 \\
0\end{array}$ & $\begin{array}{l}6.6 \\
4\end{array}$ & $\begin{array}{l}1.0 \\
0\end{array}$ & $\begin{array}{l}1.0 \\
6\end{array}$ \\
\hline P5X P7 & 0.97 & 1.09 & $\begin{array}{l}107 \\
23\end{array}$ & $\begin{array}{l}104 . \\
73\end{array}$ & 2.87 & 2.64 & 0.96 & 1.09 & $\begin{array}{l}10 \\
0\end{array}$ & $\begin{array}{l}10 \\
0\end{array}$ & 14.80 & 14.96 & $\begin{array}{l}6.3 \\
0\end{array}$ & $\begin{array}{l}6.4 \\
7\end{array}$ & $\begin{array}{l}1.0 \\
0\end{array}$ & $\begin{array}{l}1.0 \\
6\end{array}$ \\
\hline P5X P8 & 1.30 & 1.52 & $\begin{array}{l}109 . \\
90\end{array}$ & $\begin{array}{l}113 . \\
40\end{array}$ & 3.20 & 3.45 & 1.23 & 1.36 & $\begin{array}{l}10 \\
0\end{array}$ & $\begin{array}{l}10 \\
0\end{array}$ & 16.80 & 16.96 & $\begin{array}{l}6.0 \\
0\end{array}$ & $\begin{array}{l}6.1 \\
7\end{array}$ & $\begin{array}{l}1.6 \\
0\end{array}$ & $\begin{array}{l}1.7 \\
6\end{array}$ \\
\hline P6X P7 & 0.90 & 0.98 & $\begin{array}{l}103 . \\
32\end{array}$ & $\begin{array}{l}105 . \\
82\end{array}$ & 3.07 & 2.84 & 0.92 & 0.79 & $\begin{array}{l}10 \\
0\end{array}$ & $\begin{array}{l}10 \\
0\end{array}$ & 12.60 & 13.06 & $\begin{array}{l}6.4 \\
0\end{array}$ & $\begin{array}{l}6.3 \\
8\end{array}$ & $\begin{array}{l}1.0 \\
0\end{array}$ & $\begin{array}{l}0.8 \\
4\end{array}$ \\
\hline P6X P8 & 1.04 & 1.16 & $\begin{array}{l}105 . \\
23\end{array}$ & $\begin{array}{l}106 . \\
73\end{array}$ & 3.39 & 3.62 & 1.12 & 1.25 & $\begin{array}{l}10 \\
0\end{array}$ & $\begin{array}{l}10 \\
0\end{array}$ & 11.80 & 12.26 & $\begin{array}{l}6.1 \\
0\end{array}$ & $\begin{array}{l}5.9 \\
5\end{array}$ & $\begin{array}{l}1.2 \\
0\end{array}$ & $\begin{array}{l}1.3 \\
6\end{array}$ \\
\hline P7X P8 & 1.60 & 1.72 & $\begin{array}{l}114 . \\
10\end{array}$ & $\begin{array}{l}110 . \\
60\end{array}$ & 2.29 & 2.06 & 1.20 & 1.07 & $\begin{array}{l}70 . \\
3\end{array}$ & $\begin{array}{l}67 . \\
7\end{array}$ & 11.60 & 11.76 & $\begin{array}{l}6.3 \\
0\end{array}$ & $\begin{array}{l}6.1 \\
8\end{array}$ & $\begin{array}{l}1.1 \\
0\end{array}$ & $\begin{array}{l}1.0 \\
2\end{array}$ \\
\hline $\begin{array}{l}\text { LSD } \\
(5 \%)\end{array}$ & 0.20 & 0.24 & 1.46 & 1.94 & 0.29 & 0.21 & 0.15 & 0.17 & $\begin{array}{l}2.2 \\
5\end{array}$ & $\begin{array}{l}2.9 \\
5\end{array}$ & 1.03 & 1.06 & $\begin{array}{l}0.2 \\
8\end{array}$ & $\begin{array}{l}0.3 \\
2\end{array}$ & $\begin{array}{l}0.0 \\
7\end{array}$ & $\begin{array}{l}0.0 \\
9\end{array}$ \\
\hline
\end{tabular}




\section{- Genetic study of horticultural characteristics}

A great attempts were exerted to exploit the heterosis in various traits that lead to high yield to detect the best cross which use as F1 hybrid. If hybrids have great heterosis, they have valuable opportunities to detect favorable lines in consecutive generations as compared to hybrids having less heterotic impacts (Sharif et al., 2001).

Data obtained on the horticultural characteristics of 28 melon hybrids in the two seasons of 2016 and 2017 are presented in Tables 7 and 8 .

\section{- Days to picking}

With regard to heterosis, 18 out of the 28 evaluated hybrids exhibited significant negative heterosis over mid-parent for days to picking, 2 of the evaluated hybrids were $0 \%$ and 8 were positive heterosis. Meanwhile, regard to heterosis over better parent, only 12 out of the 28 evaluated hybrids exhibited significant negative heterosis and the other heterosis values between $0 \%$ to $8 \%$. The potence ratio estimates indicated over dominance towards the late parents, since their values were found more than one $(\mathrm{P}>1)$ in 12 evaluated hybrids, on the other hand, 10 hybrids (most of them which including P5) had negative values of potence ratio, this indicated partial or over dominance towards the early parents.
- Total yield The results showed that 20 hybrids of 28 evaluated hybrids gave positive values of mid parent heterosis, All studied hybrids exhibited negative mid, better. On the other hand, 11 hybrids gave positive values of better parent heterosis.

The results indicated that the potence ratio for 17 produced hybrids were between 0.07-9.69 indicating partial and over dominance towards the high parent for total yield. On the other hand, 9 hybrids exhibited partial dominance and 2 hybrids over dominance towards the lowest parent.

\section{- Early yield}

The results also indicated that the potence ratio for 8 produced hybrids were higher than 1 indicating over dominance for this character towards the high parent, 4 of hybrids were lower than 1 indicating partial dominance towards the higher parent. On the other hand, 7 hybrids gave partial dominance toward the lower parent and 9 hybrid gave over dominance toward the lower parent.

Most hybrids had negative values of mid parent heterosis, mid parent heterosis of P3X P5 was significantly the lowest in early yield/plant (-39\%), 11 hybrids had positive values of mid parent heterosis, mid parent heterosis of P1X P2 was significantly highest in early yield/plant (207\%). Also, 19 hybrids had negative values of better parent heterosis. On the contrast, 8 hybrids had positive values of better parent heterosis and one hybrid was $0 \%$.

Table 7. Quantitative genetic parameters of days to picking, total yield, early yield and average fruit weight in melon genotypes

\begin{tabular}{|c|c|c|c|c|c|c|c|c|c|c|c|c|}
\hline \multirow[b]{2}{*}{ Genotypes } & \multicolumn{3}{|c|}{ Days to picking } & \multicolumn{3}{|c|}{ Total yield } & \multicolumn{3}{|c|}{ Early yield } & \multicolumn{3}{|c|}{ Average fruit weight } \\
\hline & $\mathrm{P}$ & $\mathrm{MPH}$ & $\mathrm{BPH}$ & $\mathrm{P}$ & MPH & $\mathrm{BPH}$ & $\mathrm{P}$ & $\mathrm{MPH}$ & $\mathrm{BPH}$ & $\mathrm{P}$ & $\mathrm{MPH}$ & $\mathrm{BPH}$ \\
\hline P1X P2 & 1.03 & $-7 \%$ & $0 \%$ & 12.47 & $33 \%$ & $30 \%$ & 2.07 & $207 \%$ & $53 \%$ & 2.08 & $17 \%$ & $27 \%$ \\
\hline P1X P3 & -3.06 & $2 \%$ & $2 \%$ & 0.07 & $2 \%$ & $-19 \%$ & -0.04 & $-1 \%$ & $-25 \%$ & -0.18 & $-7 \%$ & $49 \%$ \\
\hline P1X P4 & 1.01 & $-3 \%$ & $0 \%$ & 1.16 & $20 \%$ & $2 \%$ & -0.78 & $-33 \%$ & $-53 \%$ & -0.06 & $-2 \%$ & $29 \%$ \\
\hline P1X P5 & -0.33 & $1 \%$ & $4 \%$ & 9.69 & $48 \%$ & $41 \%$ & -0.79 & $-21 \%$ & $-38 \%$ & 3.4 & $12 \%$ & $8 \%$ \\
\hline P1X P6 & 0.89 & $-3 \%$ & $0 \%$ & 1.44 & $36 \%$ & $9 \%$ & -0.77 & $-25 \%$ & $-43 \%$ & 1.29 & $6 \%$ & $11 \%$ \\
\hline P1X P7 & -9.09 & $-3 \%$ & $-2 \%$ & 6.61 & $82 \%$ & $62 \%$ & 9 & $96 \%$ & $77 \%$ & 1.67 & $28 \%$ & $53 \%$ \\
\hline P1X P8 & -2.12 & $-3 \%$ & $-2 \%$ & 0.1 & $1 \%$ & $-10 \%$ & -1.22 & $-28 \%$ & $-42 \%$ & 0.08 & $2 \%$ & $27 \%$ \\
\hline P2X P3 & 1.33 & $-9 \%$ & $-2 \%$ & 0.06 & $1 \%$ & $-18 \%$ & 1.39 & $139 \%$ & $20 \%$ & 0.35 & $11 \%$ & $87 \%$ \\
\hline P2X P4 & 1.07 & $-10 \%$ & $-1 \%$ & -0.38 & $5 \%$ & $-8 \%$ & -0.2 & $-20 \%$ & $-60 \%$ & -0.65 & $-10 \%$ & $25 \%$ \\
\hline P2X P5 & -0.23 & $3 \%$ & $2 \%$ & -3.68 & $-24 \%$ & $-17 \%$ & -0.87 & $-36 \%$ & $-23 \%$ & -1.89 & $-18 \%$ & $13 \%$ \\
\hline P2X P6 & 1.12 & $-11 \%$ & $-1 \%$ & 0.31 & $7 \%$ & $-13 \%$ & 0.09 & $9 \%$ & $-46 \%$ & 1.33 & $5 \%$ & $1 \%$ \\
\hline P2X P7 & 1.68 & $-11 \%$ & $-5 \%$ & 0.71 & $11 \%$ & $-4 \%$ & 1.13 & $113 \%$ & $6 \%$ & 1.59 & $14 \%$ & $25 \%$ \\
\hline P2X P8 & 0.7 & $-6 \%$ & $3 \%$ & -0.1 & $-1 \%$ & $-10 \%$ & 0.77 & $77 \%$ & $-12 \%$ & 0.5 & $6 \%$ & $20 \%$ \\
\hline P3X P4 & 0.01 & $0 \%$ & $3 \%$ & 0.36 & $3 \%$ & $-5 \%$ & 1.47 & $19 \%$ & $7 \%$ & 1.47 & $22 \%$ & $43 \%$ \\
\hline P3X P5 & -1.32 & $4 \%$ & $6 \%$ & -0.24 & $-7 \%$ & $-29 \%$ & -9.73 & $-39 \%$ & $-42 \%$ & -1.6 & $-29 \%$ & $19 \%$ \\
\hline P3X P6 & 1.74 & $-4 \%$ & $-2 \%$ & -7.57 & $6 \%$ & $7 \%$ & 24.33 & $25 \%$ & $27 \%$ & -0.76 & $-26 \%$ & $12 \%$ \\
\hline P3X P7 & 8.79 & $-2 \%$ & $-2 \%$ & -0.09 & $-3 \%$ & $-29 \%$ & -1.04 & $-22 \%$ & $-35 \%$ & -0.57 & $-13 \%$ & $12 \%$ \\
\hline P3X P8 & 1.09 & $-1 \%$ & $0 \%$ & 0.18 & $2 \%$ & $-9 \%$ & 3.27 & $27 \%$ & $18 \%$ & 0.06 & $1 \%$ & $25 \%$ \\
\hline P4X P5 & -22.71 & $4 \%$ & $5 \%$ & 1.35 & $29 \%$ & $6 \%$ & -1.26 & $-22 \%$ & $-33 \%$ & 1.69 & $46 \%$ & $100 \%$ \\
\hline P4X P6 & 5.76 & $-2 \%$ & $-2 \%$ & 1.31 & $11 \%$ & $2 \%$ & 1.55 & $19 \%$ & $6 \%$ & -0.65 & $-13 \%$ & $9 \%$ \\
\hline P4X P7 & -1.62 & $5 \%$ & $8 \%$ & 0.87 & $25 \%$ & $-3 \%$ & -1.15 & $-38 \%$ & $-54 \%$ & -1.94 & $-15 \%$ & $-8 \%$ \\
\hline P4X P8 & 0.07 & $0 \%$ & $2 \%$ & -3.55 & $-14 \%$ & $-10 \%$ & -1.18 & $-25 \%$ & $-38 \%$ & 1.4 & $6 \%$ & $11 \%$ \\
\hline P5X P6 & 6.09 & $-1 \%$ & $-1 \%$ & 0.23 & $7 \%$ & $-18 \%$ & -5.57 & $-28 \%$ & $-32 \%$ & 1.67 & $13 \%$ & $23 \%$ \\
\hline P5X P7 & 0.74 & $-2 \%$ & $1 \%$ & 3.17 & $24 \%$ & $15 \%$ & -0.84 & $-14 \%$ & $-27 \%$ & 0.54 & $11 \%$ & $39 \%$ \\
\hline P5X P8 & -1 & $2 \%$ & $3 \%$ & -0.31 & $6 \%$ & $-10 \%$ & -0.45 & $-2 \%$ & $-6 \%$ & -1.86 & $43 \%$ & $86 \%$ \\
\hline P6X P7 & 2.12 & $-6 \%$ & $-3 \%$ & -0.23 & $-8 \%$ & $-33 \%$ & -1.04 & $-23 \%$ & $-37 \%$ & -0.3 & $-4 \%$ & $10 \%$ \\
\hline P6X P8 & 1.94 & $-3 \%$ & $-1 \%$ & -1.36 & $-17 \%$ & $-26 \%$ & -1.64 & $-15 \%$ & $-23 \%$ & 0.47 & $7 \%$ & $27 \%$ \\
\hline P8X P7 & -1.9 & $2 \%$ & $4 \%$ & -0.79 & $-20 \%$ & $-36 \%$ & 1 & $13 \%$ & $0 \%$ & 14.71 & $47 \%$ & $52 \%$ \\
\hline
\end{tabular}


Table 8 . Quantitative genetic parameters of TSS, flesh thickness, netting and shape index in melon genotypes

\begin{tabular}{|c|c|c|c|c|c|c|c|c|c|c|c|c|}
\hline & \multicolumn{3}{|c|}{ TSS } & \multicolumn{3}{|c|}{ Flesh thickness } & \multicolumn{3}{|c|}{ Netting } & \multicolumn{3}{|c|}{ Shape index } \\
\hline Genotypes & $\mathrm{P}$ & $\mathrm{MPH}$ & $\mathrm{BPH}$ & $\mathrm{P}$ & $\mathrm{MPH}$ & $\mathrm{BPH}$ & $\mathrm{P}$ & MPH & $\mathrm{BPH}$ & $\mathrm{P}$ & MPH & $\mathrm{BPH}$ \\
\hline P1X P2 & 1.87 & $8 \%$ & $4 \%$ & -0.52 & $-11 \%$ & $-25 \%$ & 0 & $0 \%$ & $0 \%$ & 0 & $-9 \%$ & $0 \%$ \\
\hline P1X P3 & 0.32 & $3 \%$ & $-5 \%$ & 1 & $0 \%$ & $0 \%$ & -0.1 & $-10 \%$ & $-55 \%$ & 0.07 & $-21 \%$ & $20 \%$ \\
\hline D1Y P1 & 82.3 & & & & & & & & & & & \\
\hline РІХ P4 & 3 & $11 \%$ & $11 \%$ & 7 & $1 \%$ & $1 \%$ & 0 & $0 \%$ & $0 \%$ & 0 & $0 \%$ & $0 \%$ \\
\hline P1X P5 & 0.78 & $8 \%$ & $-2 \%$ & 1.28 & $4 \%$ & $1 \%$ & 1 & $100 \%$ & $0 \%$ & 0 & $11 \%$ & $0 \%$ \\
\hline P1X P6 & 1.65 & $26 \%$ & $9 \%$ & 1.87 & $7 \%$ & $3 \%$ & 1 & $100 \%$ & $0 \%$ & 0.08 & $5 \%$ & $300 \%$ \\
\hline P1X P7 & 0.96 & $8 \%$ & $0 \%$ & 9 & $3 \%$ & $3 \%$ & 1 & $100 \%$ & $0 \%$ & 0 & $-5 \%$ & $0 \%$ \\
\hline P1X P8 & 0.3 & $3 \%$ & $-6 \%$ & -0.26 & $-1 \%$ & $-6 \%$ & 0.48 & $48 \%$ & $-26 \%$ & 0 & $-15 \%$ & $0 \%$ \\
\hline P2X P3 & -0.26 & $-1 \%$ & $-5 \%$ & -0.25 & $-5 \%$ & $-21 \%$ & 0.79 & $79 \%$ & $-11 \%$ & -0.07 & $-26 \%$ & $-25 \%$ \\
\hline P2X P4 & 4.53 & $20 \%$ & $15 \%$ & 0.58 & $12 \%$ & $-7 \%$ & 0 & $0 \%$ & $0 \%$ & 0 & $11 \%$ & $0 \%$ \\
\hline P2X P5 & 0.64 & $11 \%$ & $1 \%$ & -0.26 & $-5 \%$ & $-8 \%$ & 1 & $100 \%$ & $0 \%$ & 0.2 & $9 \%$ & $0 \%$ \\
\hline P2X P6 & 0.69 & $14 \%$ & $-5 \%$ & 0.35 & $8 \%$ & $-12 \%$ & 1 & $100 \%$ & $0 \%$ & 0.07 & $0 \%$ & $100 \%$ \\
\hline P2X P7 & 1.28 & $16 \%$ & $3 \%$ & -0.11 & $-2 \%$ & $-18 \%$ & 1 & $100 \%$ & $0 \%$ & 0.05 & $10 \%$ & $-100 \%$ \\
\hline P2X P8 & -3.27 & $16 \%$ & $11 \%$ & 1.27 & $20 \%$ & $4 \%$ & 0.18 & $18 \%$ & $-41 \%$ & 0 & $-8 \%$ & $0 \%$ \\
\hline P3X P4 & -0.91 & $-8 \%$ & $-16 \%$ & 1 & $1 \%$ & $0 \%$ & -1.36 & $136 \%$ & $118 \%$ & 0.07 & $-21 \%$ & $20 \%$ \\
\hline P3X P5 & -0.47 & $-9 \%$ & $-24 \%$ & 0.33 & $1 \%$ & $-2 \%$ & -1 & $57 \%$ & $262 \%$ & 0.07 & $-16 \%$ & $20 \%$ \\
\hline P3X P6 & 1.1 & $27 \%$ & $2 \%$ & 1 & $3 \%$ & $0 \%$ & 1 & $57 \%$ & $262 \%$ & -0.09 & $37 \%$ & $-26 \%$ \\
\hline P3X P7 & 0.63 & $11 \%$ & $-5 \%$ & -3 & $-2 \%$ & $-3 \%$ & -1 & $57 \%$ & $262 \%$ & 0.03 & $-21 \%$ & $11 \%$ \\
\hline P3X P8 & 2.43 & $-1 \%$ & $-1 \%$ & -0.67 & $-3 \%$ & $-8 \%$ & -1.45 & $61 \%$ & $177 \%$ & 0.31 & $11 \%$ & $167 \%$ \\
\hline P4X P5 & 0.1 & $1 \%$ & $-9 \%$ & 1.11 & $3 \%$ & $0 \%$ & -1 & $100 \%$ & $0 \%$ & 0.1 & $22 \%$ & $-100 \%$ \\
\hline P4X P6 & 0.33 & $5 \%$ & $-9 \%$ & 1.83 & $7 \%$ & $3 \%$ & -1 & $100 \%$ & $0 \%$ & -0.06 & $-3 \%$ & $220 \%$ \\
\hline P4X P7 & 1.82 & $15 \%$ & $6 \%$ & 29 & $5 \%$ & $5 \%$ & -1 & $100 \%$ & $0 \%$ & 0.05 & $11 \%$ & $-100 \%$ \\
\hline P4X P8 & 0.95 & $-9 \%$ & $-17 \%$ & 0.15 & $1 \%$ & $-4 \%$ & -1 & $100 \%$ & $0 \%$ & 0.09 & $33 \%$ & $-50 \%$ \\
\hline P5X P6 & 3.13 & $16 \%$ & $10 \%$ & 1 & $1 \%$ & $0 \%$ & 0 & $0 \%$ & $0 \%$ & -0.02 & $5 \%$ & $33 \%$ \\
\hline P5X P7 & 3.86 & $10 \%$ & $7 \%$ & 0.5 & $2 \%$ & $-2 \%$ & 0 & $0 \%$ & $0 \%$ & -0.05 & $0 \%$ & $100 \%$ \\
\hline P5X P8 & 2.32 & $46 \%$ & $22 \%$ & 0.11 & $1 \%$ & $-6 \%$ & 1 & $19 \%$ & $0 \%$ & 0.33 & $23 \%$ & $-400 \%$ \\
\hline P6X P7 & -1.45 & $-11 \%$ & $-18 \%$ & 0.6 & $2 \%$ & $-2 \%$ & 0 & $0 \%$ & $0 \%$ & 0.03 & $0 \%$ & $100 \%$ \\
\hline P6X P8 & -0.16 & $-4 \%$ & $-23 \%$ & 0.2 & $2 \%$ & $-6 \%$ & 1 & $19 \%$ & $0 \%$ & 0.07 & $-8 \%$ & $43 \%$ \\
\hline P8X P7 & 0.22 & $4 \%$ & $-11 \%$ & 2.2 & $10 \%$ & $5 \%$ & 0.85 & $-16 \%$ & $4 \%$ & -0.04 & $-15 \%$ & $-33 \%$ \\
\hline
\end{tabular}

$\mathrm{MPH}=$ Mid parent heterosis, $\mathrm{BPH}=$ Bitter parent heterosis, $\mathrm{P}=$ potence ratio

\section{- Average fruit weight}

The results indicated that the potence ratio of 11 produced hybrids were higher than 1 indicating over dominance and 6 hybrids were lower than 1 indicating partial dominance of this character towards the heavy parent. On the contrary, 4 hybrids gave over dominance towards the light parent and the other hybrids showed partial dominance towards the light parent. The mid parent heterosis of 17 hybrids were positive and ranged from $1 \%$ to $47 \%$ and 11 hybrids were negative and ranged from $-29 \%$ to $-2 \%$. On the other hand, the better parent heterosis, all hybrids (except P4X P7) were positive.

\section{Total solids soluble (TSS)}

The results indicated that the potence ratio for 11 produced hybrids were more than one $(1<)$ indicating over dominance towards the high parent for TSS and 11 hybrids were less than one (1>) indicating partial dominance towards the high parent for this trait . On the other hand, 4 hybrids exhibited partial dominance and 2 hybrids over dominance towards the lowest parent. The results showed that 21 hybrids of 28 evaluated hybrids gave positive values of mid parent heterosis and 7 hybrids gave negative values of mid parent heterosis. On the other hand, 12 hybrids gave positive values, 14 gave negative values and 2 hybrids gave 0 of better parent heterosis.

\section{- Flesh thickness}

The results also indicated that the potence ratio for 13 produced hybrids were higher than 1 indicating over dominance for this character towards the high parent, 8 of hybrids were lower than 1 indicating partial dominance towards the higher parent. On the other hand, 9 hybrid were indicating partial and one hybrid was indicating over dominance toward the lower parent.

Most hybrids (20 hybrids) had positive values of mid parent heterosis, mid parent heterosis of P2X P8 was significantly the highest in flesh thickness (20\%). Seven hybrids had negative values of mid parent heterosis, mid parent heterosis of P1X P2 was significantly the lowest in flesh thickness $(-11 \%)$, and one hybrid gave $0 \%$. Also, 15 hybrids had negative values of better parent heterosis. On the contrast, 8 hybrids had positive values of better parent heterosis and 5 hybrid were $0 \%$.

\section{- Netting}

The results also indicated that the potence ratio for 6 produced hybrids were $(=0)$ indicating no dominance for this character, 8 of hybrids were $(=1)$ indicating complete dominance towards the higher parent and 8 of hybrids were lower than (1>) indicating partial 
dominance towards the higher parent. On the other hand, 7 hybrid were (=-1) indicating complete dominance towards the lower parent and two hybrids were less than 1- indicating over dominance toward the lower parent. Most hybrids (20 hybrids) had positive values of mid parent heterosis, mid parent heterosis of P3X P4was significantly the highest in netting (136\%), two hybrids had negative values of mid parent heterosis and 6 hybrids gave $0 \%$. On the contrast, 18 hybrids gave 0 values of better parent heterosis, 6 hybrids had positive values of better parent heterosis and 4 had negative values of better parent heterosis.

\section{- $\quad$ Shape index}

The results indicated that the potence ratio for 7 produced hybrids were $0(=0)$ indicating no dominance for this character, and 15 of hybrids were lower than1(1>) indicating partial dominance towards the higher parent. On the other hand, 6 hybrid were more than -1 indicating partial dominance towards the lower parent. Most hybrids (12 hybrids) had positive values of mid parent heterosis, 12 hybrids had negative values of mid parent heterosis and 4 hybrids gave $0 \%$. On the contrast, 8 hybrids gave 0 values of better parent heterosis, 12 hybrids had positive values of better parent heterosis and 8 had negative values of better parent heterosis.

\section{Conclusion}

It is worthy here to mention that, the inbred lines P5, P6 and P7 are resistant to powdery mildew and good horticultural characteristics. They were the best and promising inbred lines. So it might be recommended to be evaluated on a large scale and continue the breeding program to produce commercial hybrids.

\section{References}

Barros, A.K.A., G.H. Sousa, M.A. Queiróz, L.P. Elaíne Welk and J.H.C. Filho. (2011). Diallel analysis of yield and quality traits of melon fruits. Crop Breed. Appl. Biotech., 11: 313-319.

Burger, Y., U. Sa'ar, A. Distelfeld and N. Katzir. (2003). Development of sweet melon (Cucumis melo) genotypes combining high sucrose and acid content organic. J. Amer. Soc. Hort. Sci., 128(4):537- 540

Chandha, S.; J. Kumarand Vidyasagar. (2001). Combining ability over environments in tomato. Ind. J. Agric. Res., 35(3):171-175.

Chaudhary, B. R. and S. Pandey. (2010). Breeding of F1 hybrids in muskmelon: Accomplishment and prospects. Indian Journal of Arid Horticulture 5 (12): $1-5$.
Dhaliwal, M.S. (1997). Heterosis breeding in muskmelon-are view. Agricultural Reviews. 18 (1):35-42.

EL- Sayed A. A., S.H. Mahmoud and R. S. R. ElMohamedy. (2019). Breeding some cantaloupe inbred lines for resistantce to powdery mildew. Bioscience Research, 16(1): 777-792.

Falconer, D.S. and T.F.C. MacKay. (1996). Introduction to quantitative genetics. $4^{\text {th }}$ edition. Longman Green, Harlow, Essex, UK.

Feyzian, E., H. Dehghani, A.M. Rezai, M. JalaliJavaran. (2009). Diallel cross analysis for maturity and yield related traits in melon (Cucumis melo L.). Euphytica, 168: 215-223.

Floris, E., and J. M. Alvarez. (1991). Inheritance of resistance to phaerotheca fuliginea in two local melon cultivars. Acta Hort. 488, 119-124.

Gomez, A.K. and A.A. Gomez. (1984). Statistical Procedures for Agricultural Research. 2nd ed. John Wiley \& Sons Pub., New York.

James, W. C. (1971). A mannual of assessment keys for plant disease. Canada Dep. Agric. Publication No. 1458.

Jeffrey, C. A, (1980). Review of the Cucurbitaceae. Biol. J. Linn. Soc., 81, 233-247.

José, M.A.; E. Iban, A. Silviaand A Pere(2005). Inheritance mode of fruit traits in melon: Heterosis for fruit shape and its correlation with genetic distance. Euphytica, 144, 31-38.

Kadanapuradadoddi T. S., M. Pitchaimuthu, D. P. Kumar and R. D. Rawal. (2011). Heterosis and combining ability for resistance to powdery mildew in adult melon plants. Plant Breeding 130, 383 - 387.

Kenigsbuch, D., and Y. Cohen. (1989). Independent inheritance of resistance to race 1 and 2 of Sphaerotheca fuliginea in muskmelon. Plant Dis. 73, 206-208.

Munger, H.M. (1942). The possible utilization of first generation muskmelon hybrid and an improved method of hybridization. Proc. Amer. Soc. Hort. Sci., 40: 405-410.

Nunes, G.H.S., J.J. Santos Júnior, F.V. Andrade, F. Bezerra Neto, J.B. Menezes and E.W.L. Pereira. (2005). Desempenho de híbridos do grupo inodorus em Mossoró. Horticultura Brasileira, 23: 90-94.

Pandey, S.; M. Rai and B. Singh. (2005). Genetic variability and character

association in muskmelon (Cucumis melo L.). Indian J. Plant Genet. Resour., 18 (2):212-216.

Perchepied, L., M. Bardin, C. Dogimont, and M. Pitrat (2005). Relationship between loci conferring downy mildew and powderymildew resistance in melon assessed by quantitative trait locimapping. Phytopathology 95, 556-565.

Rashidi, M. and K. Seyfi. (2007). Classification of fruit shape in cantaloupe using the analysis of geometrical attributes. World Journal of Agricultural Sciences 3 (6): 735-740. 
Robinson, R. W.; H. M. Munger; T. W. Whitaker and G.W. Bohn. (1976). Genes for cucurbitaceae. Horticultural Science. 11:554-568.

Selim M.A.M.. (2019). Heterosis and combining ability for some fruit Quality traits of egyptian melon inbred lines using Line $\times$ tester analysis Egypt. J. Agric. Res., 97 (1), 317-342.

Sharif, A.; A. Bakhsh; M. Arshad; A.M. Haqqani and S. Najma. (2001). Identification of genetically superior hybrids in chickpea (Cicer arietinum L.). Pak. J. Bot., 33(4): 403- 409.
Singh, R. S. (1987): Diseases of Vegetable Crops. Oxford and IBH Publication, New Delhi, India.Wheeler, B. E., 1969: An Introduction to Plant Diseases. John Wiley and Sons, London.

,Smith, H. H. (1952). Fixing transgressive vigor in Nicotiana rustica. pdgs. 161-74 en Gowen, J.W. (ed.), Heterosis, Iowa State Coll., Ames, Iowa, EE. UU.

Wheeler, B. E. (1969). An Introduction to Plant Diseases. John Wileyand Sons, London.

$$
\begin{aligned}
& \text { تربية الكنتالوب لمقاومة البياض الاقيقي ويعض الصفات الثمرية } \\
& \text { أيمن محمد عبدربه 1، أثنرف عبداله حامد } 1 \text { ، محمود محمد رمضان } 2
\end{aligned}
$$

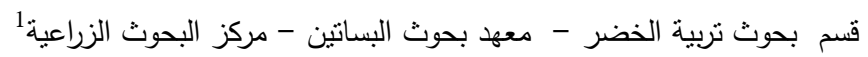

$$
\begin{aligned}
& \text { قسم آفات ووقاية نبات- الشعبة الزراعية- مركز البحوث الزراعية }
\end{aligned}
$$

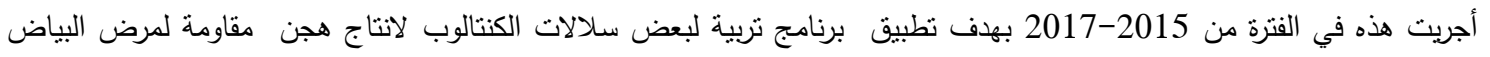

$$
\begin{aligned}
& \text { الدقيقي للحد من الخسائر في الجودة والإنتاج لمحصول الكنتالوب. نم استخدام } 8 \text { سلالة من الكنتالوب مرباة تربية داخلية عن طريق معهد بحوث } \\
& \text { البساتين. تم اجراء تلقيح نصف دائري بين السلالات لانتاج } 28 \text { هجين. نم تقييم الهجن الناتجة (28 هجن) مع ابائها (8 سلالات) واظهرت النتائج }
\end{aligned}
$$

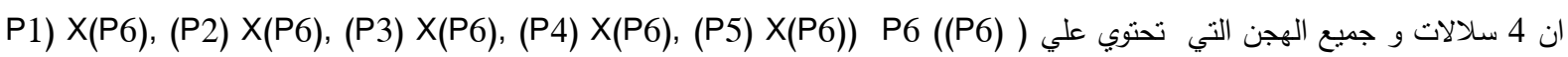

$$
\begin{aligned}
& \text { 8P5X P7, P5X P8 and P7X P بالاضافة الت عالية المقاومة للبياض الدقيقي. وانشارت النتائج ايضا } \\
& \text { ان اغلب الهجن اظهرت قوة هجين لمتوسط الاباء تجاه الاب المقاوم, وعلي العكس كانت النتائج لقوة الهجين للاب الافضل حيث كانت تتجه للاب } \\
& \text { الاقل مقاومة.كما أظهرت درجة التوريث لأغلب الهجن سيادة فائقة لصفة المقاومة للبياض الدقيقي. } \\
& \text { النتائج اظهرت ايضا وجود اختلافات كبيرة في عدد ايام الجمع, المحصول المبكر , المصصول الكلي, متوسط وزن الثرة, شكل الثرة, سمك } \\
& \text { اللحم, الثبكية و السكريات الذائبة الكلية. كما لوحظ ايضا الاختلافات المعنوية لكلا من قوة الهجين لمتوسط الاباءو افضل اب في كل الصفات. } \\
& \text { واظهرت النتائج ان السلالات P5, P6 , P7 كانت هي الافضل في المقاومة للبياض الدقيقي و الصفات البستانية, لذا يمكن استخدامها } \\
& \text { في برنامج نربية متقدم لانتاج هجن تجارية مقاومة للبياض الدقيقي. }
\end{aligned}
$$

